\title{
RELAÇÃO ENTRE ESTADO NUTRICIONAL, CONSUMO ALIMENTAR E RISCO CARDIOVASCULAR EM ADOLESCENTES DE UM MUNICÍPIO DO RIO GRANDE DO SUL ${ }^{1}$
}

\author{
RELATIONSHIP BETWEEN NUTRITIONAL STATE, \\ FOOD CONSUMPTION AND CARDIOVASCULAR RISK IN \\ ADOLESCENTS IN A MUNICIPALITY OF RIO GRANDE DO SUL
}

\author{
Mariângela Pelegrini Borsato ${ }^{2}$ e Patricia Fassina ${ }^{3}$
}

\section{RESUMO}

O sobrepeso na adolescência tende a permanecer na vida adulta e representa vulnerabilidade ao desenvolvimento de doenças crônicas. O objetivo neste estudo foi avaliar a relação entre estado nutricional, consumo alimentar e risco cardiovascular de adolescentes de um município do Rio Grande do Sul. Trata-se de um estudo quantitativo de corte transversal, com 140 estudantes, de ambos os sexos, de 10 a 15 anos incompletos, os quais responderam um questionário estruturado e registraram dois recordatórios alimentares. Foram avaliados peso, altura, circunferência da cintura e, a partir destes, calculada a Relação Cintura/Estatura (RCEst) para avaliação do risco cardiovascular. O estado nutricional dos adolescentes foi avaliado no software WHO AnthroPlus. O consumo alimentar foi avaliado no software Dietwin ${ }^{\circledR}$ Plus. Para análise estatística, foi utilizado o software Statistical Package for the Social Sciences (SPSS) versão 22.0., sendo os resultados considerados significativos a um nível de significância máximo de $5 \%(\mathrm{p} \leq 0,05)$. A eutrofia foi prevalente $(67,1 \%)$, seguida de sobrepeso $(15,7 \%)$ e obesidade $(12,9 \%)$. Verificou-se que a maioria não apresentou risco cardiovascular (75,7\%) pela RCEst. Entretanto, entre os adolescentes que apresentaram risco cardiovascular pela RCEst, constatou-se que este esteve associado àqueles com sobrepeso e obesidade $(p \leq 0,001)$. Ainda, quanto ao consumo alimentar, verificou-se que o consumo de lipídios tanto em quilocalorias como em percentual diário foi significativamente superior para aqueles que apresentaram risco cardiovascular $(\mathrm{p}=0,026 ; \mathrm{p}=0,029$, respectivamente). Diante das evidências do presente estudo, conclui-se que houve associação significativa de casos de sobrepeso e obesidade com o risco cardiovascular e com a ingestão elevada de lipídios.

Palavras-chave: Razão Cintura-Estatura, Doenças cardiovasculares, Obesidade.

\section{ABSTRACT}

Overweight in adolescence tends to remain in adulthood and represents vulnerability to the development of chronic diseases. The aim of this study was to evaluate the relationship between nutritional status, food consumption and cardiovascular risk of adolescents in a city in Rio Grande do Sul. It was a quantitative cross-sectional study, with 140 students, of both sexes, of 10 incomplete 15 years, where they answered a structured questionnaire, and recorded two food records. They were evaluated by weight, height and waist circumference. The nutritional status of the adolescents was assessed using the WHO AnthroPlus software. The Waist / Height Ratio (WHtR) was calculated. Food consumption was assessed using the Dietwin ${ }^{\circledR}$ Plus software. The results were considered significant at a maximum significance level of $5 \%(p \leq 0.05)$ and the

\footnotetext{
${ }^{1}$ Trabalho de Conclusão de Curso.

${ }^{2}$ Acadêmica do Curso de Pós-graduação em Dietoterapia nos Ciclos da Vida - Universidade do Vale do Taquari - Univates. E-mail: mariangelaborsato@hotmail.com

${ }^{3}$ Orientadora Patricia Fassina do Curso de Pós-graduação em Dietoterapia nos Ciclos da Vida - Universidade do Vale do Taquari - Univates. E-mail: patriciafassina@univates.br
} 
software used was Statistical Package for the Social Sciences (SPSS) version 22.0. Eutrophy was prevalent $(67.1 \%)$, followed by overweight, considering the sum of overweight and obesity (30.0\%). The majority did not present cardiovascular risk (75.7\%). It was found that the age group between 12 and 13 years was associated with thinness, while between 10 and 11 years was associated with obesity $(p=0.010)$. the classification of the $W H t R$ with cardiovascular risk was associated with overweight $(p \leq 0.001)$. It was found that BMI values were significantly higher for adolescents who had cardiovascular risk due to the CERR ( $p \leq 0.001)$. The consumption of lipids was significantly higher for adolescents with CERs at cardiovascular risk. There was a significant association of cases of overweight with cardiovascular risk and with a high intake of lipids.

Keywords: Waist-Height Ratio, Cardiovascular diseases, Obesity.

\section{INTRODUÇÃO}

A adolescência, segundo a Organização Mundial da Saúde (OMS), envolve a faixa etária que vai de 10 a 19 anos de idade (BRASIL, 2018). Essa fase da vida é caracterizada por diversas alterações físicas e comportamentais, em que múltiplos fatores, entre eles fisiológicos, psicológicos, sociais, econômicos e culturais, influenciam nas escolhas e nos hábitos alimentares que formarão a identidade desses indivíduos na vida adulta (PEREIRA; PEREIRA; ANGELIS-PEREIRA, 2017).

Desde há algum tempo, o Brasil vem vivenciando uma fase de transição nutricional, marcada pela substituição de refeições preparadas em casa por alimentos industrializados, prontos para o consumo e caracterizados pelo alto teor de sódio, açúcar e calorias (BRASIL, 2013). Os dados da Pesquisa de Orçamentos Familiares (POF, 2017-2018), realizada pelo Instituto Brasileiro de Geografia e Estatística (IBGE), revelaram que o consumo de alimentos in natura ou minimamente processados no total calórico da disponibilidade alimentar foi menor no Sul e Sudeste do País, se comparado ao Norte e Nordeste, e que, de modo inverso, a participação de alimentos ultraprocessados no total calórico foi maior no Sul e Sudeste no comparativo com Norte e Nordeste (IBGE, 2020), sendo que, em crianças e adolescentes, destaca-se o alto consumo de bebidas açucaradas, como refrigerantes e refrescos em pó (SOUZA; CADETE, 2017).

Esse fator impacta de forma negativa no estado nutricional dessa população. Verifica-se, de forma cada vez mais frequente, em adolescentes, alta prevalência de obesidade e sobrepeso, caracterizando um distúrbio nutricional atual marcado pelo elevado consumo de alimentos ultraprocessados (PINHO et al., 2014). De acordo com o relatório público do Sistema Nacional de Vigilância Alimentar e Nutricional (SISVAN), os dados de maio de 2019 indicaram que, no Brasil, a prevalência de sobrepeso em adolescentes era de 18,44\%, 8,29\% de obesidade e 4,39\% de obesidade grave. Já no Estado do Rio Grande do Sul, esse índice estava maior, sendo representado por 21,82\% dos adolescentes em estado de sobrepeso, 9,08\% em obesidade e 4,22\% com obesidade grave (BRASIL, 2019).

Mesmo que na fase da adolescência aconteça o pico da velocidade máxima de crescimento e o aumento das necessidades de energia, a ingestão dos alimentos de alta densidade energética pode contribuir 
para a ocorrência de sobrepeso e obesidade (ENES; SLATER, 2010). Além dos comportamentos alimentares não saudáveis, o excesso de peso dos adolescentes também está relacionado, comumente, à inatividade física e, ainda, à presença de sobrepeso e obesidade dos pais (CHAE et al., 2017). Esse determinante pode constituir um fator de risco, independente do sexo (MASCARENHAS et al., 2013).

O sobrepeso e a obesidade na adolescência tendem a permanecer na vida adulta e representam estados nutricionais vulneráveis ao desenvolvimento de doenças crônicas, como diabetes, cardiopatias, ateroscleroses, hipertensão arterial e doenças cardiovasculares para o indivíduo adulto (MARTINS et al., 2017; GUIMARÃES JUNIOR et al., 2018). Entretanto cabe ressaltar que essas doenças, usualmente manifestadas em adultos, estão sendo cada vez mais diagnosticadas em crianças e adolescentes (GUIMARÃES; GUIMARÃES, 2014; GUIMARÃES JÚNIOR et al., 2018). Ainda, o sobrepeso na infância constitui um fator desencadeante para o início do processo aterosclerótico, constituindo uma idade chave para a reversão do estado nutricional (GUIMARÃES; GUIMARÃES, 2014).

Frente ao atual cenário, o excesso de peso torna-se uma prioridade para o campo das políticas públicas, de caráter especial para a área de alimentação e nutrição, fortalecendo a urgência de se adotar estratégias de educação nutricional que objetivem atingir o público alvo (YA-TING et al., 2015; ARAÚJO et al., 2017). Uma vez que os adolescentes estão na faixa etária escolar, cabe à comunidade escolar, à família e à saúde pública investir em educação nutricional, buscando prevenção e promoção de saúde. Sendo de fácil acesso, o ambiente escolar torna-se um dos canais mais efetivos e propícios para a realização dessas atividades (MORAES; DIAS, 2012).

Com a ascensão dos índices de sobrepeso e obesidade, a prevenção e o tratamento, caso já estejam diagnosticados, desde a infância e a adolescência, podem amenizar problemas de saúde na vida adulta. $\mathrm{O}$ excesso de peso geralmente está relacionado ao acúmulo de gordura abdominal, o qual está diretamente ligado ao risco cardiovascular. Em vista disso, a avaliação do estado nutricional, do risco cardiovascular e do consumo alimentar dos adolescentes torna-se indispensável para que os fatores de risco cardiovascular sejam avaliados e identificados, uma vez que seus sintomas, nessa faixa etária, são raros, o que prejudica a detecção da evolução dos riscos de futuros eventos cardiovasculares (FONTANA; GIANNINI, 2014) .

A avaliação do estado nutricional, realizada por meio do Índice de Massa Corporal (IMC), e a análise do risco cardiovascular, realizada por meio da relação cintura/estatura (RCEst), consistem em medidas simples, rápidas, não invasivas e de baixo custo. A vantagem desse índice é que se trata de um ponto de corte único, que pode ser usado em idades maiores de cinco anos e em ambos os sexos, pois é ajustado pela estatura (ASHWELL; HSIEH, 2005).

O consumo alimentar pode ser avaliado por meio do recordatório alimentar, o qual constitui um método simples, também de baixo custo, que favorece a análise da qualidade e quantidade dos alimentos. Segundo Marchioni, Slater e Fisberg (2004), a avaliação do consumo alimentar tem como objetivo 
estimar se a ingestão de alimentos está adequada ou inadequada e identificar hábitos inadequados e/ou a ingestão excessiva de alimentos com pobre conteúdo nutricional.

Diante desse contexto, objetivou-se, neste estudo, avaliar a relação entre o estado nutricional, o consumo alimentar e o risco cardiovascular de adolescentes matriculados nas escolas municipais e estaduais de um município do Rio Grande do Sul.

\section{MATERIAIS E MÉTODOS}

Trata-se de um estudo quantitativo de corte transversal, realizado entre setembro e dezembro de 2018. Foram incluídos 140 estudantes de ambos os sexos, na faixa etária de 10 a 15 anos incompletos, matriculados nas Escolas Municipais de Ensino Fundamental (EMEFs) e na Escola Estadual de Ensino Médio (EEEM) de um município do Rio Grande do Sul. Foram excluídos os indivíduos com déficit de compreensão, os que não estiveram presentes na data da avaliação antropométrica na escola, os que apresentavam alguma patologia ou síndrome que lhes impossibilitasse a aferição do peso e da altura e os que não preencheram o questionário ou o preencheram de forma incompleta.

Os pais ou responsável legal pelos adolescentes assinaram o Termo de Consentimento Livre e Esclarecido (TCLE) como forma de consentir a participação do adolescente no estudo. Após a aprovação, os voluntários assinaram o Termo de Assentimento Livre e Esclarecido (TALE) em concordância em participar do presente estudo.

A coleta de dados aconteceu em duas etapas. Primeiramente, os sujeitos da pesquisa responderam a um questionário estruturado, não validado, desenvolvido pela pesquisadora, contendo questões de identificação, incluindo iniciais do nome, sexo, data de nascimento, série e turno da aula, além do registro do consumo alimentar, o qual foi realizado por meio de dois recordatórios alimentares, referentes a um dia habitual da semana e um dia do fim de semana, de forma voluntária.

Na segunda etapa, os voluntários do estudo foram avaliados fisicamente por meio de medidas antropométricas de peso, altura e circunferência da cintura (CC). Para aferição do peso, foi utilizada

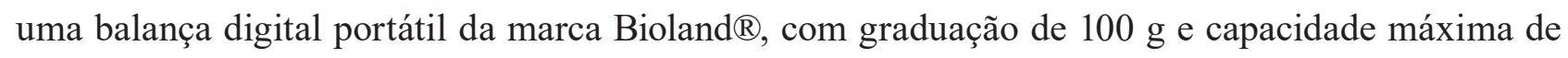
$200 \mathrm{~kg}$. O participante foi posicionado no centro da balança, descalço, com o mínimo de roupa possível, ereto com os pés juntos e os braços estendidos ao longo do corpo, mantendo-se nessa posição até que a leitura fosse realizada (BRASIL, 2011).

A medida da altura foi aferida em posição ortostática, com utilização do estadiômetro portátil da marca Sanny Profissional ${ }^{\circledR}$, com precisão de $0,1 \mathrm{~cm}$ e extensão máxima de dois metros, estando o indivíduo descalço, com a cabeça livre de adereços, ereto, com os braços estendidos ao longo do corpo, as pernas paralelas formando um ângulo reto com os pés e a cabeça erguida, olhando para um ponto fixo na altura dos olhos, posicionada no plano de Frankfurt (BRASIL, 2011). 
A CC foi aferida com o aluno em pé, em posição ereta, utilizando-se uma fita métrica flexível e inextensível, marca Cardiomed ${ }^{\circledR}$, com precisão de uma casa decimal. Essa medida foi realizada no plano horizontal, na parte mais estreita entre a crista ilíaca e a última costela. Esse ponto pode ser considerado o lugar que reflete com mais precisão o tecido adiposo visceral (WANG et al., 2003). A fita foi posicionada com firmeza em torno do local do corpo a ser medido, sem esticar, evitando-se assim a compressão do tecido subcutâneo. A leitura foi feita no centímetro mais próximo, entre uma expiração e uma inspiração.

A avaliação do estado nutricional dos adolescentes foi realizada no software WHO AnthroPlus. Os indicadores utilizados para a avaliação do estado nutricional foram as curvas de crescimento da OMS (2007) de Índice de Massa Corporal (IMC) para idade (IMC/I) e estatura para idade (E/I), os quais foram classificados de acordo com os pontos de cortes de IMC/I e E/I (BRASIL, 2011).

A Relação Cintura/Estatura (RCEst) foi calculada dividindo-se a medida da cintura $(\mathrm{cm})$ pela estatura (cm). O ponto de corte utilizado foi de 0,50 para ambos os sexos. Os adolescentes que apresentaram a RCEst igual ou maior que 0,50 foram classificados com risco cardiovascular e abaixo de 0,50, sem risco cardiovascular. Esse valor foi proposto como limite a ser empregado no diagnóstico do excesso de gordura abdominal (ASHWELL; HSIEH, 2005).

O consumo alimentar foi avaliado pelo registro dos recordatórios alimentares no software Dietwin ${ }^{\circledR}$ Plus. As refeições realizadas foram classificadas em refeições principais e lanches, sendo que as principais incluíram a soma do café da manhã, almoço e jantar e foram classificadas como uma, duas ou três refeições principais. Os lanches incluíram a soma daqueles realizados durante o período da manhã e da tarde, sendo classificados como um lanche, dois lanches ou não fez. Além da contabilização das refeições, os recordatórios alimentares foram avaliados em relação ao consumo energético total diário e de macronutrientes, considerando o teor de carboidratos, proteínas e lipídeos.

Os dados coletados foram digitados no programa Microsoft ${ }^{\circledR}$ Office Excel 2007. Os dados foram analisados por meio de tabelas, estatísticas descritivas e pelos testes estatísticos: Teste de Associação Exato de Fisher, Teste não-paramétrico Kruskal-Wallis e Teste não-paramétrico Mann-Whitney. Os resultados foram considerados significativos a um nível de significância máximo de $5 \%(\mathrm{p} \leq 0,05)$, e o software utilizado para esta análise foi o Statistical Package for the Social Sciences (SPSS) versão 22.0.

O projeto de pesquisa foi autorizado pelo Secretário de Educação do município para ser realizado e posteriormente, o projeto foi aprovado pelo Comitê de Ética em Pesquisa da Univates (COEP), sob parecer consubstanciado número 2.718.966.

\section{RESULTADOS}

A maioria dos adolescentes era do sexo masculino $(57,1 \% ; n=80)$, com faixa de idade entre 12 e $13 \operatorname{anos}(45,0 \% ; n=63)$ e frequentavam a escola no turno da manhã (73,6\%;n=103). Analisando o 
estado nutricional, a eutrofia foi prevalente $(67,1 \%$; $=94)$, seguida de excesso de sobrepeso $(15,7 \%)$ e obesidade (12,9\%). Quanto ao risco cardiovascular classificado pela RCEst, a maioria dos adolescentes não apresentou risco $(75,7 \%$; n=106). Em relação ao consumo alimentar referente ao número de refeições diárias realizadas, a maioria referiu fazer as três principais refeições $(65,0 \%$; $=91)$ (Tabela 1).

Tabela 1 - Caracterização dos adolescentes em relação às variáveis de sexo, idade, estado nutricional, risco cardiovascular e consumo alimentar referente ao número de refeições

\begin{tabular}{lcc}
\hline \multicolumn{1}{c}{ Variável } & Categoria & n (\%) \\
\hline \multirow{2}{*}{ Sexo } & Feminino & $60(42,9)$ \\
& Masculino & $80(57,1)$ \\
Faixa de Idade (anos) & $10-11$ & $29(20,7)$ \\
& $12-13$ & $63(45,0)$ \\
Turno & $14-15$ & $48(34,3)$ \\
& Manhã & $103(73,6)$ \\
Classificação IMC & Tarde & $37(26,4)$ \\
& Magreza & $4(2,9)$ \\
Classificação RCEst & Eutrofia & $94(67,1)$ \\
& Sobrepeso & $22(15,7)$ \\
& Obesidade & $18(12,9)$ \\
Lanches & Obesidade grave & $2(1,4)$ \\
& Sem risco & $106(75,7)$ \\
& Com risco & $34(24,3)$ \\
& 1 refeição & $10(7,1)$ \\
& 2 refeições & $39(27,9)$ \\
& 3 refeições & $91(65,0)$ \\
& 1 lanche & $67(47,9)$ \\
& 2 lanches & $65(46,4)$ \\
& Não fez & $8(5,7)$ \\
\hline
\end{tabular}

IMC: Índice de Massa Corporal; RCEst: Relação Cintura-Estatura; No: Número; \%: Percentual. Fonte: as autoras

Na Tabela 2, constam as estatísticas descritivas para as variáveis quantitativas de caracterização da amostra em relação aos valores mínimo e máximo, bem como à média e desvio padrão (DP), mostrando que a média de idade foi de $12,7 \pm 1,4$ anos. O peso médio dos adolescentes foi de $52,3 \pm 13,7$ $\mathrm{kg}$, a média da altura foi de $157,1 \pm 10,8 \mathrm{~cm}$ e o IMC médio atingiu $21 \pm 4,1 \mathrm{~kg} / \mathrm{m}^{2}$. A classificação média

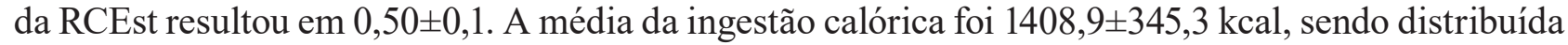
em $49,3 \pm 7,8 \%$ de carboidratos, $17,7 \pm 3,6 \%$ de proteínas e $33 \pm 6,3 \%$ de lipídios.

Tabela 2 - Caracterização dos adolescentes em relação às variáveis quantitativas de idade, estado nutricional, risco cardiovascular e consumo alimentar em relação aos valores mínimo e máximo, bem como à média e desvio padrão

\begin{tabular}{lccc}
\hline \multicolumn{1}{c}{ Variável } & Média $( \pm \mathbf{D P})$ & Mínimo & Máximo \\
\hline Idade $(\mathrm{anos})$ & $12,7( \pm 1,4)$ & 10,0 & 15,0 \\
\hline Peso $(\mathrm{Kg})$ & $52,3( \pm 13,7)$ & 26,4 & 98,9 \\
\hline Altura $(\mathrm{cm})$ & $157,1( \pm 10,8)$ & 132,0 & 187,0 \\
\hline IMC $(\mathrm{kg} / \mathrm{m} 2)$ & $21,0( \pm 4,1)$ & 14,7 & 34,0
\end{tabular}




\begin{tabular}{lccc} 
CC $(\mathrm{cm})$ & $72,7( \pm 10,9)$ & 54,0 & 108,0 \\
\hline RCEst & $0,5( \pm 0,1)$ & 0,4 & 0,8 \\
\hline VET $(\mathrm{kcal})$ & $1408,9( \pm 345,3)$ & 752,0 & 2680,4 \\
\hline CHO (KCAL) & $692,5( \pm 195,8)$ & 302,7 & 1315,7 \\
CHO $(\%)$ & $49,3( \pm 7,8)$ & 23,4 & 70,0 \\
\hline PTN (KCAL) & $246,8( \pm 78,1)$ & 122,7 & 840,4 \\
PTN (\%) & $17,7( \pm 3,6)$ & 11,3 & 31,4 \\
\hline LIP (KCAL) & $469,6( \pm 166,1)$ & 164,6 & 1214,2 \\
LIP $(\%)$ & $33,0( \pm 6,3)$ & 15,4 & 51,1 \\
\hline
\end{tabular}

DP: Desvio Padrão; IMC: Índice de Massa Corporal; CC: Circunferência da Cintura; RCEst: Relação Cintura-Estatura; VET: Valor Energético Total; CHO: Carboidratos; KCAL: Quilocalorias; PTN: Proteínas; LIP: Lipídios; \% - Percentual.

Fonte: as autoras

Na relação das variáveis sexo, idade, turno escolar, RCEst e de consumo alimentar como estado nutricional classificado pelo IMC, verificou-se que a classificação da RCEst sem risco cardiovascular foi associada ao estado nutricional de eutrofia e com risco cardiovascular associado a sobrepeso e obesidade $(\mathrm{p} \leq 0,001)$ (Tabela 3$)$.

Tabela 3 - Relação das variáveis de sexo, idade, turno escolar, risco cardiovascular pela relação cintura/estatura e de consumo alimentar referente ao número de refeições como estado nutricional classificado pelo Índice de Massa Corporal.

\begin{tabular}{|c|c|c|c|c|c|c|}
\hline \multirow{3}{*}{ Variável } & \multirow{3}{*}{ Categoria } & \multicolumn{4}{|c|}{ Classificação IMC } & \multirow{3}{*}{$\mathbf{p}$} \\
\hline & & \multicolumn{2}{|c|}{ Eutrofia } & \multicolumn{2}{|c|}{ Sobrepeso/Obesidade } & \\
\hline & & n & $\%$ & n & $\%$ & \\
\hline \multirow{2}{*}{ Sexo } & Feminino & 37 & 39,4 & 21 & 50,0 & 0,265 \\
\hline & Masculino & 57 & 60,6 & 21 & 50,0 & \\
\hline \multirow{3}{*}{ Faixa de idade (anos) } & $10-11$ & 17 & 18,1 & 12 & 28,6 & 0,385 \\
\hline & $12-13$ & 43 & 45,7 & 16 & 38,1 & \\
\hline & $14-15$ & 34 & 36,2 & 14 & 33,3 & \\
\hline \multirow{2}{*}{ Turno } & Manhã & 72 & 76,6 & 29 & 69,0 & 0,398 \\
\hline & Tarde & 22 & 23,4 & 13 & 31,0 & \\
\hline \multirow{2}{*}{ RCEst } & Sem risco & 89 & 94,7 & 13 & 31,0 & $\leq 0,001$ \\
\hline & Com risco & 5 & 5,3 & 29 & 69,0 & \\
\hline \multirow{3}{*}{ Refeições principais } & 1 refeição & 6 & 6,4 & 4 & 9,5 & 0,871 \\
\hline & 2 refeições & 27 & 28,7 & 11 & 26,2 & \\
\hline & 3 refeições & 61 & 64,9 & 27 & 64,3 & \\
\hline \multirow{3}{*}{ Lanches } & 1 lanche & 41 & 43,6 & 22 & 52,4 & 0,396 \\
\hline & 2 lanches & 46 & 48,9 & 19 & 45,2 & \\
\hline & Não fez & 7 & 7,4 & 1 & 2,4 & \\
\hline
\end{tabular}

Teste de Associação Exato de Fisher. IMC - Índice de Massa Corporal; RCEst - Relação Cintura Estatura. *Para esta análise os casos de sobrepeso e obesidade foram agrupados, porém excluídos os indivíduos classificados como magreza, devido ao número insuficiente de casos encontrados na amostra.

Fonte: as autoras

$\mathrm{Na}$ comparação das variáveis de idade, estado nutricional diagnosticado pelo IMC e consumo alimentar referente à ingestão energética, de carboidratos, proteínas e lipídeos com o risco cardiovascular classificado pela RCEst, constatou-se que os valores de IMC foram significativamente superiores 
para os adolescentes que apresentaram risco cardiovascular pela RCEst $(\mathrm{p} \leq 0,001)$. O consumo de lipídeos tanto em quilocalorias $\mathrm{COMO}$ em percentual diário foi significativamente superior para aqueles classificados como RCEst com risco cardiovascular ( $p=0,026 ; p=0,029$, respectivamente) (Tabela 4).

Tabela 4 - Comparação das variáveis de idade, estado nutricional pelo Índice de Massa Corporal e consumo alimentar referente à ingestão energética, de carboidratos, proteínas e lipídeos com o risco cardiovascular classificado pela relação cintura/estatura.

\begin{tabular}{lccccc}
\hline \multicolumn{1}{c}{ Variável } & Classificação RCEst & n & Média & Desvio Padrão & P \\
\hline \multirow{2}{*}{ Idade } & Sem risco & 106 & 12,76 & 1,26 & 0,251 \\
& Com risco & 34 & 12,32 & 1,61 & \\
\multirow{2}{*}{ IMC } & Sem risco & 106 & 19,42 & 2,78 & $\leq 0,001$ \\
& Com risco & 34 & 26,06 & 3,44 & \\
VET & Sem risco & 106 & 1425,00 & 344,04 & 0,251 \\
& Com risco & 34 & 1358,58 & 349,66 & \\
CHO KCAL & Sem risco & 106 & 690,16 & 187,83 & 0,992 \\
& Com risco & 34 & 699,89 & 221,83 & \\
CHO\% & Sem risco & 106 & 48,66 & 7,42 & 0,131 \\
& Com risco & 34 & 51,36 & 8,82 & \\
PTN KCAL & Sem risco & 106 & 250,98 & 83,36 & 0,379 \\
& Com risco & 34 & 233,58 & 57,97 & \\
PTN \% & Sem risco & 106 & 17,72 & 3,60 & 0,915 \\
& Com risco & 34 & 17,51 & 3,60 & \\
\hline \multirow{2}{*}{ LIP KCAL } & Sem risco & 106 & 483,87 & 165,17 & 0,026 \\
& Com risco & 34 & 425,12 & 163,36 & \\
\hline \multirow{2}{*}{ LIP \% } & Sem risco & 106 & 33,62 & 6,02 & 0,029 \\
& Com risco & 34 & 31,13 & 6,90 & \\
\hline
\end{tabular}

Teste Não-paramétrico Mann- Whitney. RCEst: Relação Cintura-Estatura; IMC: Índice de Massa Corporal;

VET: Valor Energético Total; CHO:Carboidratos; PTN: Proteínas; LIP: Lipídeos;

KCAL: quilocalorias; \%: percentual.

Fonte: as autoras

\section{DISCUSSÃO}

O estado nutricional classificado pelo IMC dos adolescentes avaliados no presente estudo foi relacionado, em sua maioria, à eutrofia seguida de excesso de peso, considerando os casos de sobrepeso e obesidade, assemelhando-se ao estudo de Madruga, Silva e Adami (2016), porém com um $\mathrm{n}$ amostral bem maior, realizado com 1030 adolescentes entre 10 e 17 anos, pertencentes a escolas municipais de um município do interior do Rio Grande do Sul, Brasil, em que 29,6\% dos adolescentes apresentavam excesso de peso (sobrepeso/obesidade) e a maioria dos participantes, $68,3 \%$, foi classificada como eutrófica, de acordo com o IMC. O estudo de Carneiro et al. (2014) também apresentou resultado semelhante ao presente estudo, ao avaliar 148 adolescentes em São Paulo-SP, Brasil, no qual $35,8 \%$ da amostra estavam acima do peso e a maioria, $64,2 \%$, era eutrófica.

Já um estudo realizado em Cracóvia, Polônia, por Klimek-Piotrowska et al. (2015), também com um n amostral bem maior, com 970 adolescentes poloneses, demonstrou prevalência de excesso 
de peso igual a 14,4\%, resultado inferior ao estudo presente, devido à adiposidade em adolescentes da Cracóvia ainda ser menor do que em outras sociedades desenvolvidas que lutam contra a epidemia da obesidade, apesar de ter aumentado durante a última década (KLIMEK-PIOTROWSKA et al., 2015). Comparando o atual estudo a um estudo realizado por Malinski e Voser (2016), no qual foram avaliados 427 alunos, matriculados em três escolas distintas, sendo uma da rede privada e duas da rede pública de ensino da cidade de Porto Alegre-RS, Brasil, foi encontrada uma ocorrência de 43,8\% de indivíduos acima do peso, representando valores superiores aos encontrados no presente estudo. Supõe-se que esse fato possa ter ocorrido por se tratarem de escolas de um grande centro urbano, enquanto o público alvo do presente estudo localiza-se em uma cidade do interior.

Com o processo de industrialização e urbanização, houve o aumento da ingestão de alimentos calóricos e a diminuição da atividade física, estabelecendo o princípio do sobrepeso, com acúmulo de gordura corporal. A população infantojuvenil, em áreas urbanas, tem poucos espaços gratuitos para praticar atividades físicas e incorporam formas de lazer sedentárias, fazendo uso de computadores e televisão (TARDIDO; FALCÃO, 2006). Em contrapartida, os habitantes da área rural têm mais acesso a frutas e verduras, além de atividades de lazer diferentes dos habitantes da área urbana, que geram maior gasto de energia, como atividades de esportes e corridas. Por esse motivo, as crianças residentes da área rural teriam menor incidência de sobrepeso e obesidade. Nos achados de Baggio, Marques e Mendes (2014), foi evidenciada maior prevalência de obesidade na área urbana, devido à realização das refeições fora de casa, ao alto consumo de alimentos calóricos e ricos em gorduras, sal e açúcar, juntamente com o sedentarismo e o aumento da urbanização.

De acordo com a Pesquisa Nacional de Saúde dos Escolares (PeNSE), realizada em 2015, os resultados indicaram que o estado nutricional dos adolescentes escolares do Brasil caracteriza-se por baixas prevalências de déficit de peso e alta prevalência de excesso de peso (IBGE, 2016), assim como observado no atual estudo. A crescente questão da obesidade infantil pode ser retardada se a sociedade se concentrar nas causas. Existem muitas causas para a obesidade infantil, algumas mais cruciais que outras. Uma dieta balanceada e intervenção de atividade física conduzida na comunidade com um componente escolar é mais eficaz na prevenção da obesidade. Também, se os pais aplicarem um estilo de vida mais saudável em casa, muitos problemas de obesidade podem ser evitados (SAHOO et al., 2015).

$\mathrm{Na}$ avaliação do risco cardiovascular diagnosticado pela relação cintura/estatura (RCEst), no estudo observacional transversal realizado por Madruga, Silva e Adami (2016), os autores observaram que 18,3\% dos adolescentes avaliados apresentaram risco cardiovascular, índice inferior ao comparado no estudo presente, em que $24,3 \%$ apresentaram risco cardiovascular. O presente estudo também apresentou prevalência de risco cardiovascular pela RCEst superior ao estudo de Teixeira et al. (2018), no qual 19,1\% dos adolescentes avaliados, com idades entre 10 e 18 anos, apresentaram risco cardiovascular referente à avaliação da RCEst. 
A RCEst e a Circunferência da Cintura (CC) têm sido indicadas como medidas antropométricas adicionais para avaliar a obesidade e adiposidade central, porque estariam associadas ao impacto adverso nos fatores de risco cardiovascular, independentemente da condição do peso corporal, sendo benéficas para identificação prévia de crianças que se favoreceriam com uma intervenção precoce (MUST; HOLLANDER; ECONOMOS, 2006). Esses parâmetros seriam melhores preditores de risco cardiovascular em crianças e adolescentes quando comparados ao IMC isoladamente (KAHN; IMPERATORE; CHENG, 2005; McCARTHY; ASHWELL, 2006).

Entretanto, caso a diminuição do IMC não ocorra, pode-se aumentar o risco do desenvolvimento de síndrome plurimetabólica (diabetes mellitus, resistência à insulina, hipertensão arterial, dislipidemia, obesidade visceral e albuminúria). Além da má qualidade de vida dos indivíduos, aumentam-se os gastos do país, efeito desse processo que será percebido diretamente na saúde pública (BIENER; CAWLEY; MEYERHOEFER, 2017). Assim, tornam-se necessárias ações que promovam uma ingestão de alimentos mais saudáveis e um aumento da prática de atividade física diária (McMAHON et al., 2017).

Na relação entre o risco cardiovascular classificado pela RCEst e o estado nutricional diagnosticado pelo IMC, os adolescentes que se apresentaram sem risco cardiovascular estavam com o IMC significativamente associado à magreza e eutrofia. Já os adolescentes com risco cardiovascular estavam associados ao IMC de sobrepeso e obesidade, de forma significativa. Assim como no estudo de Teixeira et al. (2018), os adolescentes que apresentaram RCEst elevada confirmaram maiores chances de apresentar excesso de peso. Resultados que confirmam com Ramírez-Vélez et al. (2017) e Perona et al. (2017), que avaliaram adolescentes colombianos e espanhóis, respectivamente, evidenciaram que indivíduos com avaliações acima do recomendado para RCEst podem apresentar maior produção de citoquinas, como IL-6 e TNFa no organismo (ROTH; REINEHR, 2010). Em excesso, essas proteínas podem elevar o risco do desenvolvimento de doenças ligadas à síndrome metabólica, que engloba a aterosclerose, hipertensão arterial, hiperlipidemia e diabetes mellitus (SIJTSMA et al., 2014).

De acordo com os achados do estudo atual, a maioria dos adolescentes realizava as três principais refeições mais, pelo menos, um lanche no dia. Um estudo feito entre escolares em um município, também do Rio Grande do Sul, mostrou que os escolares que relataram realizar quatro ou mais refeições por dia apresentaram 50\% menos chances de estarem acima do peso do que aqueles que realizavam até três refeições por dia (MARTINS et al., 2017). Já Rech et al. (2010), em estudo realizado na cidade de Caxias do Sul-RS, constataram que o número de refeições por dia apresentou associação contrária com a prevalência de sobrepeso e obesidade, sendo que indivíduos que relataram fazer três refeições ou menos por dia apresentaram $48 \%$ mais chances de estar em sobrepeso.

O Guia Alimentar para a População Brasileira recomenda que sejam realizadas, no mínimo, as três principais refeições, entre elas, café da manhã, almoço e jantar e, em algumas situações específicas, também é recomendado o consumo de lanches entre as refeições (BRASIL, 2014). 
O café da manhã é definido como a primeira refeição consumida pela manhã. Essa refeição é vastamente considerada como um importante componente do estilo de vida saudável, e um de seus benefícios é que a sua realização diária promove uma proteção contra o excesso de peso (COULTHARD; PALLA; POT, 2017; LAZAROU et al., 2009). O almoço é a refeição mais consumida pelos adolescentes, seguido pelo jantar, que, apesar de ter uma boa prevalência de consumo, também é a refeição em que acontecem maiores substituições de alimentos tradicionais por alimentos comumente consumidos nos lanches, como fast food, alimentos ricos em açúcar e gordura (ARAKI et al., 2011; LEAL et al., 2010).

Outro dado significativo deste estudo mostrou que a média de ingestão de calorias provenientes de gordura foi significativamente superior em crianças classificadas com risco cardiovascular por meio da RCEst, dado que demonstra que a maioria dos adolescentes, sejam eles eutróficos, sejam com sobrepeso, consumiam alimentos fontes de gordura em demasia. No estudo com escolares de Menêses et al. (2017), houve alta ingestão de alimentos hipercalóricos e ricos em sódio e gordura, oriundos de alimentos industrializados, como bolachas/biscoitos doces ou recheados, doces, balas e chocolates, batata frita, batata de pacote, salgadinhos fritos, hambúrguer, embutidos e refrigerantes, sendo a maioria consumidos no horário do lanche, em troca do jantar e nos fins de semana.

Na PeNSE de 2009, em que foi investigado o consumo regular ( $\geq 5$ dias/semana) de quatro grupos de alimentos ultraprocessados entre adolescentes, foram encontradas altas proporções de adolescentes que consumiram guloseimas, refrigerantes, biscoitos doces e embutidos (IBGE, 2009). Já na segunda edição da PeNSE, em 2012, consta prevalência de consumo regular ( $\geq 5$ dias/semana) de guloseimas, refrigerantes, biscoitos doces, salgados fritos e salgadinhos de pacote (IBGE, 2013). Ainda, no estudo realizado da PeNSE 2015, no qual foi avaliado o consumo diário de, pelo menos, um alimento ultraprocessado e, apesar de as porções, em gramas ou mililitros desses alimentos consumidas por dia não serem conhecidas, é preocupante que quatro em cada dez adolescentes brasileiros tenham relatado consumir diariamente esses alimentos (IBGE, 2015). Ressalta-se que o consumo de alimentos hipercalóricos e com altos teores de açúcar, gordura e sal estão associados à manifestação de doenças crônicas, tanto na própria infância como na vida adulta (BLOOM et al., 2015; BRASIL, 2016).

O presente estudo apresenta como pontos fortes o tamanho da amostra, visto que foram analisados quase que a totalidade dos adolescentes nessa faixa etária do município e, como limitação, a possível omissão de refeições ou o tamanho das porções nos recordatórios, por esquecimento ou por timidez. Destaca-se, também, que foi a primeira vez que um estudo que investigou a relação do estado nutricional, o risco cardiovascular e o consumo alimentar foi realizado nesta cidade. Por esta razão, torna-se necessária a realização de outros estudos, para futuro confrontamento de dados. 


\section{CONCLUSÃO}

Apesar de a maioria dos adolescentes avaliados no presente estudo ter apresentado estado nutricional de eutrofia, considerou-se uma prevalente parcela de excesso de peso, considerando os casos de sobrepeso e de obesidade na população estudada.

Quanto ao risco cardiovascular classificado pela RCEst, a maioria dos adolescentes não apresentou risco e ainda foi verificado que a classificação da RCEst sem risco cardiovascular foi associada ao estado nutricional de eutrofia e com risco cardiovascular associado ao sobrepeso e obesidade, constatando-se que os valores de IMC foram significativamente superiores para os adolescentes que apresentaram risco cardiovascular pela RCEst.

Em relação ao consumo alimentar referente ao número de refeições diárias realizadas, a maioria referiu fazer as três principais refeições do dia. Observou-se ainda que o consumo de lipídeos tanto em quilocalorias como em percentual diário foi significativamente superior para aqueles adolescentes classificados com risco cardiovascular pela RCEst. Enfim, diante das evidências do presente estudo, resume-se a associação significativa de casos de sobrepeso e obesidade com o risco cardiovascular e com uma ingestão elevada de lipídios.

Salienta-se que a implementação de medidas de intervenção no combate e na prevenção do excesso de peso em indivíduos adolescentes torna-se necessária para conter a epidemia da obesidade. Assim, considera-se de suma importância a constante educação alimentar e o acompanhamento de profissionais da saúde para a população desta faixa etária, para que o crescimento e desenvolvimento decorram de forma saudável, contribuindo para o desenvolvimento de hábitos alimentares saudáveis, a prevenção de doenças crônicas e um bom estado de saúde na idade adulta.

\section{REFERÊNCIAS}

ARAKI, E. L. et al. Padrão de refeições realizadas por adolescentes que frequentam escolas técnicas de São Paulo. Revista Paulista de Pediatria, v. 29, n. 2, p. 164-170, 2011.

ARAÚJO, A. L. et al. O impacto da educação alimentar e nutricional na prevenção do excesso de peso em escolares: uma revisão bibliográfica. Revista de Obesidade, Nutrição e Emagrecimento, v. 11, n. 62, p. 94-105, 2017.

ASHWELL, M.; HSIEH, S. D. Six reasons why the waist-to-height ratio is a rapid and effective global indicator for health risks of obesity and how its use could simplify the international public health message on obesity. International Journal of Food Sciences and Nutrition, v. 56, n. 5, p. 303-307, 2005. 
BAGGIO, A.; MARQUES, F.; MENDES, K. G.; Obesidade infanto juvenil na área urbana e rural: uma revisão sistemática. Revista Brasileira de Nutrição Clínica, v. 29, n. 1, p. 76-80, 2014.

BIENER, I. B.; CAWLEY, J.; MEYERHOEFER, C. The medical care costs of youth obesity: an instrumental variables approach. National Bureau of Economic Research, Working Paper, n. 23682, 2017.

BLOOM, M. I. T. et al. Ingestão de vitaminas e minerais em uma amostra de hipertensos de um município da região nordeste do Brasil. Revista Brasileira de Nutrição Clínica, v. 30, n. 2, p. 154-158, 2015.

BRASIL. Ministério da Saúde. Secretaria de Atenção à Saúde. Departamento de Ações Programáticas e Estratégicas. Proteger e cuidar da saúde de adolescentes na atenção básica. 2. ed. Brasília: Ministério da Saúde, 2018. 233 p. Disponível em: https://bit.ly/3klw8v8. Acesso em: 26 jul. 2020.

BRASIL. Ministério da Saúde. Secretaria de Atenção à Saúde. Departamento de Atenção Básica. Guia alimentar para a população brasileira. 2. ed., 1. reimpr. Brasília: Ministério da Saúde, 2014. 156 p. Disponível em: https://bit.ly/36jZEMQ. Acesso em: 26 jul. 2020.

BRASIL. Ministério da Saúde. Secretaria de Atenção à Saúde. Departamento de Atenção Básica. Orientações para a coleta e análise de dados antropométricos em serviços de saúde: Norma Técnica do Sistema de Vigilância Alimentar e Nutricional - SISVAN. Brasília: Ministério da Saúde, 2011. 76 p. Disponível em: https://bit.ly/3ljcjFY. Acesso em: 23 jul. 2020.

BRASIL. Ministério da Saúde. Secretaria de Atenção à Saúde. Departamento de Atenção Básica. Saúde da criança: aleitamento materno e alimentação complementar. 2. ed. Brasília: Ministério da Saúde, 2015. 184 p. Disponível em: https://bit.ly/3kdjmip. Acesso em: 26 jul. 2020.

BRASIL. Ministério da Saúde. Sistema de Vigilância Alimentar e Nutricional - SISVAN. Relatório do Estado Nutricional de adolescentes no Brasil. Brasília: Ministério da Saúde, 2019. Disponível em: https://bit.ly/35hXgqF. Acesso em: 28 de junho de 2019.

BRASIL. Ministério do Desenvolvimento Social e Combate à Fome. Educação Alimentar e Nutricional: o direito humano a alimentação adequada e o fortalecimento de vínculos familiares nos serviços socioassistenciais. Caderno de atividades. Secretaria Nacional de Segurança Alimentar e Nutricional (SESAN) e Secretaria Nacional de Assistência Social (SNAS). Brasília: Ministério do Desenvolvimento Social e Combate à Fome, 2013. Disponível em: https://bit.ly/2IkCSwb. Acesso em: 23 jul. 2020. 
CARNEIRO, I. B. P. et al. Antigos e novos indicadores antropométricos como preditores de resistência à insulina em adolescentes. Arquivos Brasileiros de Endocrinologia \& Metabologia, v. 58, n. 8, p. 838-843, 2014.

CHAE S. M. et al. Weight control in adolescents: focus groups with Korean adolescents and their teachers. Journal of Pediatric Nursing, v. 33, p. 4-9, 2017.

COULThARD, J. D.; PALLA, L.; POT, G. K. Breakfast consumption and nutrient intakes in 4-18-yearolds: UK National Diet and Nutrition Survey Rolling Programme (2008-2012). British Journal of Nutrition, v. 118, n. 4, p. 280-290, 2017.

ENES, C. C.; SLATER, B. Obesidade na adolescência e seus principais fatores determinantes. Revista Brasileira de Epidemiologia, v. 13, n. 1, p. 163-171, 2010.

FONTANA, P. V.; GIANNINI, D. T. Associação de indicadores antropométricos com fatores de risco cardiovascular em adolescentes com excesso de peso. Adolescência e Saúde, v. 11, n. 4, p. 68-78, 2014.

GUIMARÃES, I. C. B.; GUIMARÃES, A. C. Síndrome Metabólica na infância e adolescência. Um fator maior de risco cardiovascular. Revista Baiana de Saúde Pública, v. 30, n. 2, p. 349-362, 2014.

GUIMARÃES JUNIOR, M. S. et al. Fator de risco cardiovascular: a obesidade entre crianças e adolescentes nas macrorregiões brasileiras. Revista Brasileira de Obesidade, Nutrição e Emagrecimento, v. 12, n. 69, p. 132-142, 2018.

IBGE. Instituto Brasileiro de Geografia e Estatística. Pesquisa nacional de saúde do escolar: 2009. Rio de Janeiro: IBGE, 2009. 140 p. Disponível em: https://bit.ly/3naQKYV. Acesso em: 26 jul. 2020

IBGE. Instituto Brasileiro de Geografia e Estatística. Pesquisa nacional de saúde do escolar: 2012. Rio de Janeiro: IBGE, 2013. 256 p. Disponível em: https://bit.ly/2GQrw2y. Acesso em: 26 jul. 2020.

IBGE. Instituto Brasileiro de Geografia e Estatística. Pesquisa nacional de saúde do escolar: 2015. Rio de Janeiro: IBGE, 2016. 126 p. Disponível em: https://bit.ly/35h05YV. Acesso em: 26 jul. 2020.

IBGE. Instituto Brasileiro de Geografia e Estatística. Pesquisa de orçamentos familiares 2017-2018. Rio de Janeiro: IBGE; 2020. Disponível em: https://bit.ly/3kh824L. Acesso em: 26 jul. 2020. 
KAHN, H. S.; IMPERATORE, G.; CHENG, Y. J. A population based comparison of BMI percentiles and waistto-height ratio for identifying cardiovascular risk in youth. The Journal of Pediatrics, v. 146, n. 4, p. 482-488, 2005.

KLIMEK-PIOTROWSKA, W. et al. Anthropometry and body composition of adolescents in Cracow, Poland. PLoS One, v. 10, n. 3, p. 1-12, 2015.

LAZAROU, C. et al. Dietary and other lifestyle characte,ristics of Cypriot school children: results from the nationwide CYKIDS study. BMC Public Health, v. 9, n. 1. p. 147, 2009.

LEAL, G. V. S. et al. Consumo alimentar e padrão de refeições de adolescentes, São Paulo, Brasil. Revista Brasileira de Epidemiologia, v. 13, n. 3, p. 457-467, 2010.

MADRUGA, J. G.; SILVA, F. M.; ADAMI, F. S.; Positive association between waist to height ratio and hypertension in adolescents. Revista Portuguesa de Cardiologia, v. 35, n. 9, p. 479-484, 2016.

MALINSKI, M. P.; VOSER, R. C. Sobrepeso e obesidade em jovens escolares. Arquivos de Ciências da Saúde, v. 23, n. 1, p. 68-72, 2016.

MARCHIONI, D. M.; SLATER, B.; FISBERG, R. M. Aplicação das Dietary Reference Intakes na avaliação da ingestão de nutrientes para indivíduos. Revista de Nutrição, v. 17, n. 2, p. 207-216, 2004.

MARTINS, F. S. et al. Prevalência de sintomas para transtornos alimentares, sobrepeso e obesidade em escolares do município de Bom Jesus - RS. Revista Brasileira de Obesidade, Nutrição e Emagrecimento, v. 11, n. 61, p. 31-38, 2017.

MASCARENHAS, L. P. G. et al. Influência do excesso de peso dos pais em relação ao sobrepeso e obesidade dos filhos. Pensar a prática, v. 16, n. 2, p. 320-618, 2013.

McCARTHY, H. D.; ASHWELL, M. A study of central fatness using waist-to-height ratios in UK children and adolescents over two decades supports the simple message - 'keep your waist circumference to less than half your height'. International Journal of Obesity, v. 30, n. 6, p. 988-992, 2006.

McMAHON, E. M. et al. Physical activity in European adolescents and associations with anxiety depression and well-being. European Child \& Adolescente Psychiatry, v. 26, n. 1, p. 111-122, 2017. 
MENÊSES, L. E. N. et al. Consumo alimentar e estado nutricional de crianças em uma escola privada de Palmas, Tocantins. Revista Desafios, v. 4, n. 3. p. 43-51, 2017.

MORAES, P. M. M.; DIAS, C. M. S. B. Obesidade infantil a partir de um olhar histórico sobre alimentação. Revista interação psicológica, v. 16, n. 2, p. 317-326, 2012.

MUST, A.; HOLLANDER, S. A.; ECONOMOS, C. D. Childhood obesity: a growing public health concern. Expert Review of Endocrinology \& Metabolism, v. 1, n. 2. p. 233-254, 2006.

PEREIRA, T. S.; PEREIRA, R. C.; ANGELIS-PEREIRA, M. C. A. Influência de intervenções educativas no conhecimento sobre alimentação e nutrição de adolescentes de uma escola pública. Ciência \& Saúde Coletiva, v. 22, n. 2, p. 427-435, 2017.

PERONA, J. S. et al. Waist circumference shows the highest predictive value for metabolic syndrome and waist-to-hip ratio for its components in Spanish adolescents. Nutrition Research, v. 45, n. 4, p. 38-45, 2017.

PINHO, L. et al. Excess weight and food consumption of adolescents in public schools in northern Minas Gerais state, Brazil. Ciência \& Saúde Coletiva, v. 19, n. 1, p. 67-74, 2014.

RAMÍREZ-VÉLEZ, R. et al. Using LMS tables to determine waist circumference and waist-to-height ratios in Colombian children and adolescents: the Fuprecol study. BMC Pediatrics, v. 17, n. 1, p. 1-11, 2017.

RECH, R. R. et al. Prevalência de obesidade em escolares de 7 a 12 anos de uma cidade Serrana do RS, Brasil. Revista Brasileira de Cineantropometria \& Desempenho Humano, v. 12, n. 2, p. 90-97, 2010.

ROTH, C. L.; REINEHR, T. Roles of gastrointestinal and adipose tissue peptides in childhood obesity and changes after weight loss dueto life style intervention. Archives of Pediatrics and Adolescent Medicine, v. 164, n. 2, p. 131-138, 2010.

SAHOO, K. et al. Childhoodobesity: causes and consequences. Journal of Family Medicine and Primary Care, v. 4, n. 2, p. 187-192, 2015.

SIJTSMA, A. et al. Waist-toheight ratio waist circumference and BMI as indicators of percentage fat mass and cardiometabolic risk factors in children aged 3 - 7 years. Clinical Nutrition, v. 33, n. 2, p. 311-315, 2014. 
SOUZA, A. A.; CADETE, M. M. M. O papel das famílias e da escola na formação de hábitos alimentares saudáveis de crianças escolares. Revista Pedagógica, v. 19, n. 40, p. 136-154, 2017.

TARDIDO, A. P.; FALCÃO, M. C. O impacto da modernização na transição nutricional e obesidade. Revista Brasileira de Nutrição Clínica, v. 21, n. 2, p. 117-124, 2006.

TEIXEIRA, F. et al. Prevalência de fatores antropométricos e bioquímicos sobre o estado nutricional de adolescentes. Revista Brasileira de Obesidade, Nutrição e Emagrecimento, v. 12, n. 76, p. 1067-1077, 2018.

WANG, J. et al. Comparisons of waist circumferences measured at 4 sites. The American Journal of Clinical Nutrition, v. 77, n. 2, p. 379-384, 2003.

YA-TING, C. Y. et al. Technology enhanced game based team learning for improving intake of food groups and nutritinal elements. Computers \& Education, v. 88, p. 143-159, 2015. 
